\title{
Vibronic dephasing of anharmonic molecules. II. Impurity molecules isolated in low-temperature matrices
}

\author{
D. J. Diestlera) and A. H. Zewaib) \\ Arthur Amos Noyes Laboratory of Chemical Physics, ${ }^{\text {c) }}$ California Institute of Technology, Pasadena, \\ California 91125 \\ (Received 21 December 1978; accepted 28 June 1979)
}

The quantum-mechanical theory of vibronic dephasing presented in the first paper of this series is applied to the case of a diatomic impurity dissolved in a solid rare-gas host. An explicit expression for the pure dephasing rate $T_{2}^{\prime-1}$ is derived in terms of microscopic properties of the impurity and host, and the effects of variations in the parameters characterizing these properties are investigated. The expression for $T_{2}^{\prime-1}$ is applied specifically to the system $\mathrm{Cl}_{2} / \mathrm{Ar}$ in order to relate the results to those of previous classicaltrajectory calculations and of experimental measurements. The significance of anharmonicity in the intramolecular potential curve (of the impurity) is demonstrated.

\section{INTRODUCTION}

In the first article ${ }^{1}$ of this series (hereinafter referred to as I), we considered in general terms the dephasing of optically active impurity molecules dilutely dispersed in an inert, inactive medium. Under the as sumption that the relevant optical transition (between intramolecular states of the impurity) is homogeneously broadened, we established connections between certain spectroscopic measurements and the dephasing time $T_{2}$, emphasizing that the total rate of dephasing

$$
T_{2}^{\prime-1}=T_{2}^{\prime-1}+T_{1}^{\prime-1}
$$

comprises two contributions:

(i) pure phase relaxation $\left(T_{2}^{\prime-1}\right)$, which arises from elastic scattering processes leaving the intramolecular state of the impurity unaltered;

(ii) energy relaxation $\left(T_{1}^{-1}\right)$, which is due to inelastic transitions between the intramolecular states of the impurity.

In I, we developed a rather general quantum-mechanical theory of vibronic dephasing of impurity molecules dissolved in a solid host, obtaining closed expressions for $T_{2}^{\prime}$. There we focused on the qualitative and semi quantitative aspects of the theory, exploring in particular the dependence of $T_{2}^{\prime}$ upon the nature of the intramolecular potential-energy surface (PES). The purpose of the present work is to apply the theory of I and to derive a quantitative expression for $T_{2}^{\prime}$ for molecules isolated in matrices.

In Sec. II, we shall present the model and derive a general expression for $T_{2}^{\prime}$ in terms of microscopic properties of the system. Next, in Sec. III, we shall apply our model to the system $\mathrm{Cl}_{2} / \mathrm{Ar}$, calculating $T_{2}^{\prime}$ for various vibronic transitions. We shall also explore the dependence of $T_{2}^{\prime}$ upon certain features of the PES and upon the temperature. Finally, in Sec. IV, we shall compare our results for $\mathrm{Cl}_{2} / \mathrm{Ar}$ with the computational

\footnotetext{
${ }^{2}$ Permanent address: Department of Chemistry, Purdue University, West Lafayette, IN 47907.

${ }^{b)}$ Alf red P. Sloan Fellow.

c) Contribution No. 5946 .
}

results of Shugard et al. ${ }^{2}$ and with the experimental work of Bondybey and Fletcher. ${ }^{3}$

\section{MODEL AND EXPRESSION FOR $T_{2}^{\prime}$}

The physical system of interest, i. e., a dilute solution of impurity molecules in a solid host, can be adequately represented as a single impurity occupying a substitutional site in an otherwise perfect crystal. For the case of a diatomic impurity in a monatomic host, the essential elements of the model are depicted in Fig. 1. We shall assume the orientation of the impurity to be fixed. Moreover, we shall take the total PES to be expressible as

$$
\epsilon_{i}(\mathbf{Q} ; \mathrm{u})=U_{I}^{(i)}(\mathbf{Q})+\sum_{i} U_{I l}^{(i)}(\mathbf{Q} ; \mathrm{u}),
$$

where $U_{I}^{(1)}\left[\equiv \epsilon_{i}(Q ; 0)\right]$ is the intramolecular binding potential and $U_{H}^{(1)}$ is the interaction potential between the impurity and the $l$ th lattice molecule.

Now it seems reasonable to suppose that collinear encounters, i. e., those in which the neighboring lattice atom approaches the impurity molecule along a line parallel to the principal relative atomic displacement of the relevant intramolecular normal mode, would play a dominant role in dephasing, although the possibility of significant effects arising from noncollinear collisions cannot be ruled out. Taking the $z$ axis of the coordinate system (with its origin at the center of mass of the impurity) to be parallel to the principal relative atomic displacement of the intramolecular mode (e. g. , parallel to the internuclear vector in the case of a diatomic purity), we require an expression for the $z$ component of the displacement of the $i$ th lattice molecule. For the purpose of deriving such an expression, let us assume that the lattice is pure and isotropic. Then there are three branches of acoustic normal modes: one longitudinal and two transverse. The wave vector associated with a given mode can be expressed as

$$
q=(\sin \theta \cos \phi, \sin \theta \sin \phi, \cos \theta) q,
$$

where $\theta$ and $\phi$ are the usual polar angles specifying the direction of the wave vector and $q$ is its magnitude. The normalized polarization vector associated with (the modes of) the longitudinal branch is then given by 


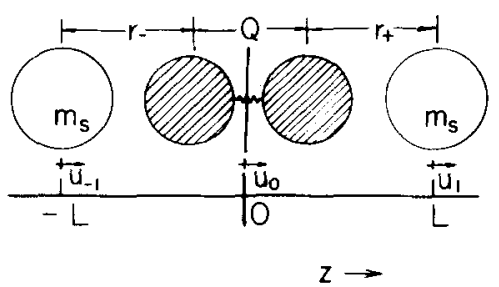

FIG. 1. Schematic of a homonuclear diatomic impurity in a substitutional site of a crystal lattice. Only the collinear (i.e., longitudinal) displacements are depicted.

$$
e_{l}=(\sin \theta \cos \phi, \sin \theta \sin \phi, \cos \theta) \text {. }
$$

Taking the polarization vector of one of the transverse branches, say $t 1$, to lie in the $x-y$ plane, we have

$$
e_{t 1}=(\sin \phi,-\cos \phi, 0)
$$

and

$$
e_{t 2}=(\cos \theta \cos \phi, \cos \theta \sin \phi,-\sin \theta) .
$$

Thus, we can express the required displacement $\mathrm{as}^{4}$

$$
\begin{aligned}
u_{t z}=\left(N m_{s}\right)^{-1 / 2}\left\{\left[\sum_{\mathrm{a} l}\left(\hbar / 2 \omega_{a l}\right)^{1 / 2} \cos \theta_{\mathrm{a} l}\right.\right. \\
\left.\quad \times \exp \left(i q_{l} R_{i} \cos \theta_{\mathrm{a}_{l}}\right) \mathrm{b}_{\mathrm{a}_{l}}+\text { h. a. }\right] \\
-\left[\sum_{\mathrm{a}_{t 2}}\left(\hbar / 2 \omega_{q_{t 2}}\right)^{1 / 2} \sin \theta_{\mathrm{a}_{t 2}}\right. \\
\left.\left.\times \exp \left(i q_{t 2} R_{i} \cos \theta_{\mathrm{a}_{t 2}}\right) \mathrm{b}_{\mathrm{a}_{t 2}}+\text { h. a. }\right]\right\},
\end{aligned}
$$

where $\omega_{\mathrm{q}}$ is the frequency associated with the normal mode of wave vector $\mathrm{q}$ and $R_{i}$ is the equilibrium distance of the (c. m. of the) ith lattice molecule from the origin; h. a. refers to the "Hermitian adjoint" of the first term; $m_{s}$ and $N$ are, respectively, the mass and total number of lattice molecules.

Comparing Eq. (II. 4) with Eq. (III. 20) of I, we can determine the coefficients $C_{k \alpha ; \nu}\left(=C_{t \varepsilon ; \alpha}\right)$ involved in $D_{\mu \nu}\left(=D_{\mathbf{q a}}\right)$ [see Eq. (III. 25) of I]. We can then substi tute the resulting expression for $D_{\mu \nu}$ into Eq. (III. 29) of I to get $T_{2}^{\prime-1}$. Doing this, we see that, in general, there are both longitudinal and transverse contributions to the pure dephasing rate and possibly also interferences between these. However, rather than account for all of these effects simultaneously in the present treatment, we shall for the moment consider only the longitudinal phonons with wave vectors lying nearly parallel to $u_{i \varepsilon}$. Then, from Eq. (II. 4), we have

$$
\begin{aligned}
u_{i z}=\left(N m_{s}\right)^{-1 / 2}\left[\sum_{\mathrm{a}_{l}}\left(\hbar / 2 \omega_{\mathrm{a}_{l}}\right)^{1 / 2}\right. & \\
& \left.\times \exp \left(i q_{l} R_{i}\right) \mathrm{b}_{\mathrm{a}_{l}}+\text { h. a. }\right],
\end{aligned}
$$

and from Eq. (III. 25) of I,

$$
\begin{aligned}
D_{\mu \nu}= & \left(\hbar / 2 N m_{s}\right)\left(\omega_{\mu} \omega_{\nu}\right)^{-1 / 2} \sum_{l} \sum_{\kappa}\left[\left\langle\bar{\chi}_{i v}^{0}\left|\epsilon_{i ; l \kappa}^{(2)}\right| \bar{\chi}_{i v}^{0}\right\rangle\right. \\
& \left.-\left\langle\bar{\chi}_{f \nu^{\circ}}^{0}\left|\epsilon_{f ; l \kappa}^{(2)}\right| \bar{\chi}_{f \nu^{\circ}}^{0}\right\rangle\right] \exp \left(-i q_{\mu} R_{l}\right) \exp \left(i q_{\nu} R_{\kappa}\right)
\end{aligned}
$$

where $l$ and $\kappa$ label the impurity $(l, \kappa=0)$ and its nearest "collinear" neighbors $(l, k= \pm 1)$, and $\mu, \nu$ label only longitudinal modes.

Note that Eq. (II. 5) corresponds to Eq. (15.6) of Ref. 4 , which expands the impurity-lattice coupling in powers of the strain $\epsilon$, in turn approximated as

$$
\epsilon=(\partial u / \partial z)_{z=0},
$$

where $u$ is given by Eq. (II. 5) with $R_{t}=z$. Clearly, the approach based on the strain neglects the effects of the transversely polarized phonons and should therefore be employed with due caution. For example, it may be that the $T^{7}$ dependence of $T_{2}^{\prime-1}$ commonly predicted for the case of the Debye solid is due to the assumption inherent in Eq. (II. 5). ${ }^{5}$

Before proceeding, we emphasize that even though the model takes into account only the "collinear" contribution to the impurity-lattice coupling, full threedimensional lattice-dynamical effects are partially (in a sense clarified by the preceding discussion) included.

Now, for the case of a symmetric (e. g. , homonuclear diatomic) impurity, the portion of the PES which depends upon $\mathbf{Q}$ can be expressed as [see Eq. (II. 1)]

$$
\epsilon_{i}(\mathbf{Q} ; u)=U_{I}^{(i)}(\mathbf{Q})+\phi_{i}\left(r_{+}\right)+\phi_{i}\left(r_{-}\right) \text {. }
$$

The coordinates $r_{+}$and $r_{-}$are defined by (see Fig. 1)

$$
\begin{aligned}
& r_{+} \equiv L+u_{1}-u_{0}+f_{+}^{i}(\mathbf{Q}), \\
& r_{-} \equiv L+u_{0}-u_{-1}+f^{i}(\mathbf{Q}),
\end{aligned}
$$

where $u_{l}$ is the ( $z$ component of the) displacement of molecule $l, L$ is the lattice constant, and $f_{ \pm}^{i}$ are functions of $Q$ only, depending upon the internal structure of the impurity. In Eq. (II. 8), $U_{I}^{(1)}$ is the intramolecular PES for the isolated impurity in the $i$ th electronic state; $\phi_{i}$ is the (assumed known) interatomic potential. Henceforth, for the sake of simplicity of notation, the indices $I$ and $i$ will be suppressed where there is no sacrifice of clarity.

Expanding the sums in Eq. (II. 6) and exploiting the symmetry displayed in Eq. (II. 8) to combine terms, we obtain

$$
\begin{aligned}
D_{\mu \nu}= & \left(\hbar / N m_{s}\right)\left(\omega_{\mu} \omega_{\nu}\right)^{-1 / 2} v_{i f} \\
& \times\left[\cos \left(q_{\mu}-q_{\nu}\right) L-\cos q_{\mu} L-\cos q_{\nu} L+1\right],
\end{aligned}
$$

where

$$
v_{i f} \equiv\left\langle\bar{\chi}_{i v}^{0}\left|\phi_{i}^{\prime \prime}\right| \bar{\chi}_{i v}^{0}\right\rangle-\left\langle\bar{\chi}_{f v^{\prime}}^{0}\left|\phi_{f}^{\prime \prime}\right| \bar{\chi}_{f \nabla^{\prime}}^{0}\right\rangle
$$

and

$$
\phi^{\prime \prime}=\phi^{\prime \prime}(\mathbf{Q}) \equiv\left(d^{2} \phi / d r_{ \pm}^{2}\right)_{r_{t}=L+f_{ \pm}(Q)} .
$$

Note that $\phi^{\prime \prime}$ is the second derivative of the intermolecular potential curve $\phi$ evaluated at the equilibrium configuration of the lattice and hence is a function only of in tramolecular coordinates $Q$ and static distances $(L)$ be tween the impurity and its nearest-neighboring lattice molecules. (For a similar discussion in connection with the strain-tensor approach to dephasing, see Ref. 4.)

Now, substituting expression (II. 10) for $D_{\mu \nu}$ into Eq. (III. 29) of I, converting the sums on $\mu$ and $\nu$ to inte- 
grals and carrying out the integration over $\omega_{\nu}$, we get

$T_{2}^{\prime-1}=\pi \hbar^{-2} \int_{0}^{\omega_{\max }} d \omega \rho^{2}(\omega)|D(\omega)|^{2} \bar{n}(\omega)[\bar{n}(\omega)+1]$,

where

$$
D(\omega) \equiv\left(4 \hbar / N m_{s} \omega\right) v_{i f} \sin ^{2}(q L / 2)
$$

and $\rho$ is the frequency distribution function having a cutoff of $\omega_{\max }$. For a Debye solid, the dispersion relation is simply

$$
q=\omega / v_{l},
$$

where the speed of longitudinal phonons is given in terms of the mass density $\rho_{m}$ by ${ }^{4}$

$$
v_{l}=\omega_{D} /\left(6 \pi^{2} \rho_{m} / m_{s}\right)^{1 / 3} .
$$

The frequency distribution function is therefore

$$
\rho(\omega)= \begin{cases}3 N \omega^{2} / \omega_{D}^{3}, & 0 \leq \omega \leq \omega_{D}, \\ 0, & \omega>\omega_{D},\end{cases}
$$

where $\omega_{D}\left(=\omega_{\max }\right)$ is the Debye cutoff frequency. Now, substituting expressions (II. 14) and (II. 17) into Eq. (II. 13) and using the relation

$$
\bar{n}(\omega)=[\exp (\beta \hbar \omega)-1]^{-1},
$$

we have

$$
\begin{aligned}
& {\left[T_{2}^{\prime}(i, f)\right]^{-1}=\left(144 \pi\left|v_{i f}\right|^{2} / m_{s}^{2} \omega_{D}^{6}\right)} \\
& \quad \times \int_{0}^{\omega_{D}} d \omega \omega^{2} \sin ^{4}\left(\omega L / 2 v_{l}\right) \exp (\beta \hbar \omega) /[\exp (\beta \hbar \omega)-1]^{2},
\end{aligned}
$$

where $\beta \equiv 1 /\left(k_{B} T\right)$. In Eq. (II. 19), we have labeled $T_{2}^{\prime}$ by the intramolecular transition $(i, f)$ to which it corresponds.

Upon making the transformation

$$
x=\beta \hbar \omega,
$$

we can recast expression (II. 19) as

$$
\begin{aligned}
{\left[T_{2}^{\prime}(i, f)\right]^{-1}=} & {\left[144 \pi\left|v_{i f}\right|^{2} /\left(m_{s}^{2} \omega_{D}^{6} \beta^{3} \hbar^{3}\right)\right] } \\
& \times \int_{0}^{x_{D}} x^{2} \sin ^{4}(\eta x) \exp (x) /[\exp (x)-1]^{2} d x,
\end{aligned}
$$

where

$$
\begin{aligned}
& x_{D} \equiv \beta \hbar \omega_{D}, \\
& \eta=L /\left(2 \hbar \beta v_{l}\right)=L k_{B} T / 2 \hbar v_{l} .
\end{aligned}
$$

At sufficiently low temperatures, we can introduce the approximation

$$
\sin (\eta x) \sim \eta x
$$

into Eq. (II. 21) to obtain

$$
\begin{aligned}
& {\left[T_{2}^{\prime}(i, f)\right]^{-1} \simeq\left[9 \pi\left|v_{i f}\right|^{2} k_{B}^{7} L^{4} /\left(m_{s}^{2} \omega_{D}^{6} \hbar^{7} v_{l}^{4}\right)\right]} \\
& \quad \times \int_{0}^{\infty} d x x^{6} \exp (x) /[\exp (x)-1]^{2} \cdot T^{7}, \quad \text { low } T .
\end{aligned}
$$

The integral in Eq. (II. 24) can be readily evaluated by parts to give

$\left[T_{2}^{\prime}(i, f)\right]^{-1}=\left[9 \pi\left|v_{i f}\right|{ }^{2} k_{B}^{7} L^{4} 6 ! \zeta(6) /\left(\omega_{D}^{6} m_{s}^{2} \hbar^{7} v_{l}^{4}\right)\right] \cdot T^{7}$, where $\zeta$ is the standard zeta function. ${ }^{6}$ Hence, in the limit of low temperature, the pure dephasing rate vanishes as $T^{7}$-a feature characteristic of a Raman phonon-scattering process that has appeared previously in several contexts. $1,4,7,8$

Let us attempt to make a connection between expressions (II. 24) and (III. 43) of I, which was derived follow ing the "strain approach." Using Eq. (II. 16) to eliminate $\omega_{D}$ from Eq. (II. 24), we obtain in the low-T limit

$\left[T_{2}^{\prime}(i, f)\right]^{-1}=\frac{6 !\left(k_{B} / \hbar\right)^{7}}{4 \pi^{3} \rho_{m}^{2} v_{l}^{10}}\left|v_{i f}\right|^{2} L^{4} T^{7}, \quad$ low $T$.

Now, equating expressions (II. 26) and (III. 43) of I, we find

$$
\left|v_{i f}\right|^{2} L^{4}=9\left|v_{f f}^{(2)}\right|^{2},
$$

if we assume that

$$
v_{l}=v_{s} \text {. }
$$

Using the definitions of $v_{i f}$ [Eq. (II. 11)] and $v_{f f}^{(2)}[\mathrm{Eq}$. (III. 33) of I] and assuming that

$$
\left|\left\langle\bar{\chi}_{i v}^{0}\left|\phi_{i}^{\prime \prime}\right| \bar{\chi}_{i v}^{0}\right\rangle\right| \ll\left|\left\langle\bar{\chi}_{f v}^{0}\left|\phi_{f}^{\prime \prime}\right| \bar{\chi}_{f v}^{0}\right\rangle\right|
$$

we have from Eq. (II. 27)

$L^{4}\left|\left\langle\bar{\chi}_{f v^{\prime}}^{0}\left|\phi_{f}^{\prime \prime}\right| \bar{\chi}_{f r^{\prime}}^{0}\right\rangle\right|^{2}=9\left|\left\langle\bar{\chi}_{f v^{\prime}}^{0}\left|\left[\left\langle\phi_{f}\left|\mathbf{V}_{2}\right| \phi_{f}\right\rangle\right]\right| \bar{\chi}_{f v^{\prime}}^{0}\right\rangle\right|^{2}$.

Thus, we conclude that

$$
\phi_{f}^{\prime \prime}(\mathbf{Q})=3 L^{-2}\left\langle\phi_{f}\left|\mathbf{V}_{2}\right| \phi_{f}\right\rangle
$$

is at least consistent with Eq. (II. 26), if the matrix elements of $\phi_{f}^{\prime \prime}$ and $V_{2}$ are taken to be real. Equation (II. 31) relates the anharmonic impurity-lattice couplings responsible for pure dephasing and hence provides a fundamental, albeit tenuous, connection between the two approaches.

\section{APPLICATION TO HOMONUCLEAR DIATOMIC IMPURITY: $\mathrm{Cl}_{\mathbf{2}} / \mathrm{Ar}$}

In this case, there is only one internal coordinate, namely, the internuclear separation $Q$ (see Fig. 1). Let us assume for the intramolecular potential curve the Morse form, ${ }^{9}$ i. e.,

$$
U^{(t)}(Q)=D_{i}\left\{\exp \left[-2 a_{i}\left(Q-Q_{0}^{i}\right)\right]-2 \exp \left[-a_{i}\left(Q-Q_{0}^{i}\right)\right]\right\} \text {. }
$$

Then the vibronic wave functions are

$\bar{\chi}_{i v}^{0}(Q)=N_{i v} \exp \left(-z_{i} / 2\right) z_{i}^{b i v} L_{v}^{\left(b_{i v}\right)}\left(z_{i}\right)$,

where

$$
\begin{aligned}
& z_{i} \equiv 2 d_{i} \exp \left[-a_{i}\left(Q-Q_{0}^{i}\right)\right], \\
& b_{i v} \equiv 2 d_{i}-2 v-1, \quad v=0,1, \ldots, \leq d_{i}-\frac{1}{2}, \\
& d_{i} \equiv\left(2 D_{i}\right)^{1 / 2} / \hbar a_{i} .
\end{aligned}
$$

In Eqs. (III. 1)-(III. 3), $L_{v}^{\left(b_{v}\right)}$ is the generalized Laguerre polynomial, ${ }^{6} N_{i v}$ is a normalization constant, $v$ labeling the vibrational levels within the $i$ th manifold; $Q_{0}^{f}$ and $\mu$ are, respectively, the equilibrium internuclear separation (in $i$ th manifold) and the reduced mass of the diatomic.

For the interatomic potentials $\phi$, we take the 


$$
\begin{aligned}
& \mathrm{LJ}(12,6) \text { form } \\
& \qquad \begin{array}{l}
10 \\
\left(r_{ \pm}\right)=4 \hat{\epsilon}\left[\left(\hat{\sigma} / r_{ \pm}\right)^{12}-\left(\hat{\sigma} / r_{ \pm}\right)^{6}\right] .
\end{array}
\end{aligned}
$$

For convenience, ${ }^{11} \phi$ is fit to the Morse form

$\phi=\Delta\left\{\exp \left[-2 \alpha\left(r_{ \pm}-r_{0}\right)\right]-2 \exp \left[-\alpha\left(r_{ \pm}-r_{0}\right)\right]\right\}$

by requiring the zeros and minima of expressions

(III. 4) and (III. 5) to coincide. This procedure yields

$$
\begin{aligned}
& \Delta=\hat{\epsilon}, \\
& r_{0}=2^{1 / 6} \hat{\sigma}, \\
& \alpha=\ln 2 /\left[\left(2^{1 / 6}-1\right) \hat{\sigma}\right] .
\end{aligned}
$$

Now, for the case of a homonuclear diatomic impurity, the functions $f_{t}$ defined by Eq. (II.9) are simply

$$
f_{+}=f_{-}=-Q / 2 \text {. }
$$

(Note that, for polyatomic impurities, $f_{t}$ can be worked out in terms of the normal coordinates. ) Hence, when the lattice displacements are set to zero,

$$
r_{ \pm}=L-\left(Q_{0}+\bar{Q}\right) / 2 \text {, }
$$

$\bar{Q}$ being the displacement of $Q$ from its equilibrium value of $Q_{0}$. Let us introduce an arbitrary displacement $\delta r$ by means of the definition

$$
L \equiv r_{0}-\delta r / 2+Q_{0} / 2 \text {. }
$$

Thus, combining Eqs. (III. 8) and (III. 9), we have from Eq. (III. 5)

$$
\begin{aligned}
\phi^{\prime \prime}(Q)= & 2 \alpha^{2} \Delta\{2 \exp [\alpha(\bar{Q}+\delta r)] \\
& -\exp [\alpha(\bar{Q}+\delta r) / 2]\} .
\end{aligned}
$$

Note that the condition $\delta r=0$ corresponds to the physical situation in which the potential energy is a minimum (at $T=0^{\circ} \mathrm{K}$ classically). Positive values of $\delta r$ corre spond to a "compressed" condition of the diatomic, i. e., to moving the nearest neighbors closer to the diatomic so that the potential energy increases.

\section{A. Details of the computations}

Using the substitution

$$
x=y x_{D}
$$

in Eq. (II. 21), we obtain the working expression for $\left[T_{2}^{\prime}(i, f)\right]^{-1}$, namely,

$$
\left[T_{2}^{\prime}(i, f)\right]^{-1}=\left(144 \pi\left|v_{i f}\right|^{2} / m_{a}^{2} \omega_{D}^{3}\right) I(T),
$$

where

$$
I(T) \equiv \int_{0}^{1} d y y^{2} \sin ^{4} \gamma y \exp \left(x_{D} y\right) /\left[\exp \left(x_{D} y\right)-1\right]^{2}
$$

and

$$
\gamma \equiv L\left(6 \pi^{2} \rho_{m} / m_{s}\right)^{1 / 3} / 2 .
$$

The computation falls naturally into two parts:

(i) determination of $v_{i f}$;

(ii) evaluation of integral $I(T)$.

From Eq. (II. 11), we see that $v_{i f}$ is a difference between matrix elements of the type $\left\langle\bar{\chi}_{i v}^{0}\left|\phi_{i}^{\prime \prime}\right| \bar{\chi}_{i v}^{0}\right\rangle=\int_{-\infty}^{\infty}\left|\bar{\chi}_{i v}^{0}(Q)\right|^{2} \phi_{i}^{\prime \prime}(Q) d Q$,

where $\bar{\chi}_{i v}^{0}$ and $\phi_{i}^{\prime \prime}$ are given by Eqs. (III. 2) and (III. 10), respectively. The required integrals were performed numerically using the extended Simpson's Rule. ${ }^{6}$ Orthonormality of the wave functions $\left(\bar{\chi}_{i v}^{0}\right)$ was maintained to at least 1 part in $10^{5}$. As an additional check, the calculation was repeated using the 64 -point Gauss-Hermite quadrature formula ${ }^{6}$ instead of Simpson's Rule. The values of $T_{2}^{\prime}$ calculated using the two different quadrature schemes agree to at least $1 \%$ in most cases.

Evaluation of $I(T)$ is facilitated by observing that the integrand is an even function of $y$. Thus,

$I(T)=\frac{1}{2} \int_{-1}^{1} d y y^{2} \sin ^{4} \gamma y \exp \left(x_{D} y\right) /\left[\exp \left(x_{D} y\right)-1\right]^{2}$

is in a form suitable for application of the standard Gaussian quadrature formula, ${ }^{6}$ the 16 -point version of which we employed. Again using Simpson's Rule, we confirmed the Gaussian quadratures.

\section{B. Results for the system $\mathrm{Cl}_{2} / \mathrm{Ar}$}

Following the approach taken in I, we have adopted two alternate viewpoints in carrying out the computations. On the one hand, we have assumed that $\phi_{f}=\phi_{f}$, i. e. , that the anharmonic impurity-lattice couplings in the initial and final electronic states are equal. On the other, we have invoked inequality (II. 29), i. e., the couplings in the two states are very different.

\section{Equal anharmonic coupling}

Using the parameters listed in Tables I and II, we have computed $T_{2}^{\prime}$ for the vibronic transitions $\left(0, v^{\prime}\right)$ $\left[X^{1} \Sigma_{g}^{+}\left(v^{\prime \prime}=0\right) \rightarrow B^{3} \pi_{u}\left(v^{\prime}=0,1,2, \ldots, 14\right)\right]$ in the sys tem $\mathrm{Cl}_{2} / \mathrm{Ar}$ at various temperatures. The interatomic LJ parameter $\hat{\epsilon}$ is taken as the geometric mean ${ }^{10}$ of those for $\mathrm{Cl}_{2}$ and $\mathrm{Ar}$; $\hat{\sigma}$ is estimated by the "recipe" described in Ref. 12. Table III gives $T_{2}^{\prime}$ as a function of $v^{\prime}$ at $4 \mathrm{~K}$, while Table IV lists $T_{2}^{\prime}$ at various temperatures for a selection of experimentally observed transitions.

Since our model is quite idealized, there is naturally some uncertainty in the parameters characterizing it,

TABLE I. Physical parameters for the system $\mathrm{Cl}_{2} / \mathrm{Ar}$.

\begin{tabular}{ll}
\hline \hline Parameter & Value \\
\hline$\mu$ & $2.943 \times 10^{-23} \mathrm{~g}(17.73 \mathrm{amu})$ \\
$m_{s}$ & $6.631 \times 10^{-23} \mathrm{~g}(39.94 \mathrm{amu})$ \\
$\hat{\epsilon}$ & $146.0 \mathrm{~cm}^{-1}\left(210^{\circ} \mathrm{K}\right)$ \\
$\hat{\sigma}$ & $2.85 \AA$ \\
$L$ & $4.06 \AA$ \\
$\rho_{m}$ & $1.40 \mathrm{~g} \mathrm{~cm}^{-3}$ \\
$T_{D}$ & $90.0 \mathrm{~K}^{\circ}$ \\
$\delta r$ & $0.0 \AA$ (at potential minimum) \\
\hline \hline
\end{tabular}


TABLE II. Parameters characterizing electronic states of the system $\mathrm{Cl}_{2} / \mathrm{Ar}$.

\begin{tabular}{lccc}
\hline \hline State & $D_{i}\left(\mathrm{~cm}^{-1}\right)^{\mathrm{a}}$ & $Q_{0}^{i}(\AA)^{\mathrm{b}}$ & $a_{i}\left(\AA^{-1}\right)^{\mathrm{a}}$ \\
\hline$X^{1} \Sigma_{\boldsymbol{z}}^{+}$ & 28177.0 & 1.988 & 1.711 \\
$B^{3} \Pi_{u}$ & 3146.4 & 2.396 & 2.398 \\
\hline \hline
\end{tabular}

${ }^{2}$ Calculated from values of $\omega_{e}$ and $\omega_{e} x_{e}$ given in Ref. 3.

brom Ref. 3 .

particularly those connected with the PES. To explore the dependence of $T_{2}^{\prime}$ upon these parameters, we have performed three sets of calculations, the results of which are arranged in three columns in Table III. All parameters given in Table I hold for the first column, labeled " $\delta r=0$." As pointed out above, $\delta r=0$ corresponds to the situation in which the diatomic resides in an absolute potential minimum (classically at $T=0^{\circ} \mathrm{K}$ ). The coupling between the lattice modes and the intramolecular vibration should be weakest in this instance. The column labeled " $\delta r=1.0 \AA$ " corresponds to the diatomic's being compressed by moving its nearest neighbors closer at equilibrium. In this case, we expect the strength of the impurity-lattice coupling to increase and hence the dephasing time to decrease. Indeed, the results bear out our expectations. An alternative way to increase the impurity-lattice coupling strength is to make the interaction potential $\phi$ "harder," i. e. , to de crease $\hat{\sigma}$. The third column (labeled " $\hat{\sigma}=1.75 \AA$ ") of Table III shows that, as we expect, $T_{2}^{\prime}$ decreases from its "normal" value, for which $\hat{\sigma}=2.85 \AA$.

In the fifth column of Table III, we give estimates of $T_{1}$ for the $\left(0, v^{\prime}=10-14\right)$ transitions. These were ob-

TABLE III. $T_{2}^{\prime}$ (psec) for the vibronic transitions $X^{1} \Sigma_{j}^{\dagger}\left(v^{\prime \prime}=0\right)$ $\rightarrow B^{3} \pi_{u}\left(v^{\prime}=0,1, \ldots, 14\right)$ for the system $\mathrm{Cl}_{2} / \mathrm{Ar}$ at $4^{\circ} \mathrm{K}$. The number in parentheses following an entry indicates the power of 10 by which it is to be multiplied.

\begin{tabular}{|c|c|c|c|c|c|}
\hline \multirow[b]{2}{*}{$v^{\prime}$} & \multicolumn{3}{|c|}{$T_{2}^{\prime 2}$} & \multirow[b]{2}{*}{$T_{1}(\delta r=0)^{b}$} & \multirow[b]{2}{*}{$T_{2}(\delta r=0)^{c}$} \\
\hline & $\delta r=0$ & $\delta r=1.0 \AA$ & $\hat{\sigma}=1.75 \AA$ & & \\
\hline 0 & $6.45(5)$ & $8.70(3)$ & $2.56(4)$ & $\cdots$ & $\cdots$ \\
\hline 1 & 3. $36(4)$ & $4.59(2)$ & $1.24(3)$ & $\cdots$ & $\cdots$ \\
\hline 2 & $9.56(3)$ & $1.31(2)$ & $3.30(2)$ & $\cdots$ & $\cdots$ \\
\hline 3 & 4. $06(3)$ & 56.3 & $1.32(2)$ & $\cdots$ & $\cdots$ \\
\hline 4 & $2.07(3)$ & 29.0 & 62.6 & $\cdots$ & $\cdots$ \\
\hline 5 & $1.17(3)$ & 16.5 & 32.8 & $\cdots$ & $\cdots$ \\
\hline 6 & $7.05(2)$ & 10.0 & 18.2 & $\cdots$ & $\cdots$ \\
\hline 7 & 4. $42(2)$ & 6.3 & 10.4 & $\cdots$ & $\cdots$ \\
\hline 8 & $2.84(2)$ & 4.1 & 6.1 & $\cdots$ & $\cdots$ \\
\hline 9 & $1.85(2)$ & 2.7 & 3.6 & $\cdots$ & $\cdots$ \\
\hline 10 & $1.21(2)$ & 1.8 & 2.1 & 14.2 & 23.0 \\
\hline 11 & 80.2 & 1.2 & 1.2 & 9.6 & 15.5 \\
\hline 12 & 52.5 & 0.8 & 0.7 & 5.6 & 9.2 \\
\hline 13 & 34.0 & 0.5 & 0.4 & 2.1 & 3.7 \\
\hline 14 & 21.6 & 0.3 & 0.2 & 1.3 & 2.3 \\
\hline
\end{tabular}

${ }^{2}$ Calculated using expression (III. 12) and the normal parameters listed in Tables I and II. Variations of these parameters are indicated at the top of the column.

'Calculated using pseudolocal mode theory (Ref. 13) of vibrational energy relaxation (see discussion in text).

cCalculated using Eq. (I. 1).
TABLE IV. T $T_{2}^{\prime}$ (psec) calculated using Eq. (III.12) as a function of temperature for selected vibronic transitions $X^{1} \Sigma_{t}^{+}\left(v^{\prime \prime}=0\right)$ $\rightarrow B{ }^{3} \pi_{u}\left(v^{\prime}=10-14\right)$ in the system $\mathrm{Cl}_{2} / \mathrm{Ar}$. Parameters are listed in Tables I and II, except that $\delta r=1.0 \AA$. The number in parentheses following an entry indicates the power of 10 by which it is to be multiplied.

\begin{tabular}{clcccc}
\hline \hline$T\left({ }^{\circ} \mathrm{K}\right)$ & $v^{\prime}=10$ & $v^{\prime}=11$ & $v^{\prime}=12$ & $v^{\prime}=13$ & $v^{\prime}=14$ \\
\hline 1 & $1.42(6)$ & $9.32(5)$ & $6.10(5)$ & $3.95(5)$ & $2.51(5)$ \\
4 & $1.16(2)$ & 76.0 & 50.0 & 33.0 & 20.0 \\
10 & 0.61 & 0.42 & 0.27 & 0.18 & 0.11 \\
20 & 0.041 & 0.027 & 0.018 & 0.011 & 0.007 \\
\hline \hline
\end{tabular}

tained using a new model ${ }^{13}$ based upon the dynamical participation of pseudolocal modes. The model assumes that the only contribution to $T_{1}$ is due to (pure) vibrational relaxation from $v^{\prime}$ to $v^{\prime}-1$ and hence neglects additional nonradiative and all radiative mechanisms. The parameters of the $T_{1}$ calculation are just those of Table $I$, although it should be noted that the temperature is taken to be $0{ }^{\circ} \mathrm{K}$ for the $T_{1}$ calculations. Since $T_{1}$ does not depend strongly upon temperature, however, we can estimate $T_{2}$ (at $4^{\circ} \mathrm{K}$ ) using Eq. (I. 1). This is done in the last column of Table III.

Table IV indicates that the dephasing time decreases (or the dephasing rate increases) rapidly with increas ing temperature. The plots in Fig. 2 provide a more graphical demonstration of this effect. Note from Eq. (III. 12) that since the dependence of $T_{2}^{\prime-1}$ on intramolecular properties is contained solely in the factor $\left|v_{i f}\right|^{2}$, the ratio $\left[T_{2}^{\prime}(i, f)\right]^{-1} /\left[T_{2}^{\prime}(i, f)\right]_{T_{1} 1}^{-1}{ }^{\circ} \mathrm{K}$ depends only on the properties of the lattice and the temperature, and hence, for a given lattice, only on the temperature. The marked increase in $T_{2}^{\prime-1}$ with $T$ is again in accord with our expectation. As $T$ increases, the population of phonons which are capable of scattering from the impurity increases and hence the effective collision rate increases. As a consequence, the rate of dephasing increases.

It should be emphasized that even though $T_{2}^{\prime}$ depends quite strongly upon the parameters $\delta r$ and $\hat{\sigma}$, its dependence on $v^{\prime}$, which is of primary interest, is in fact

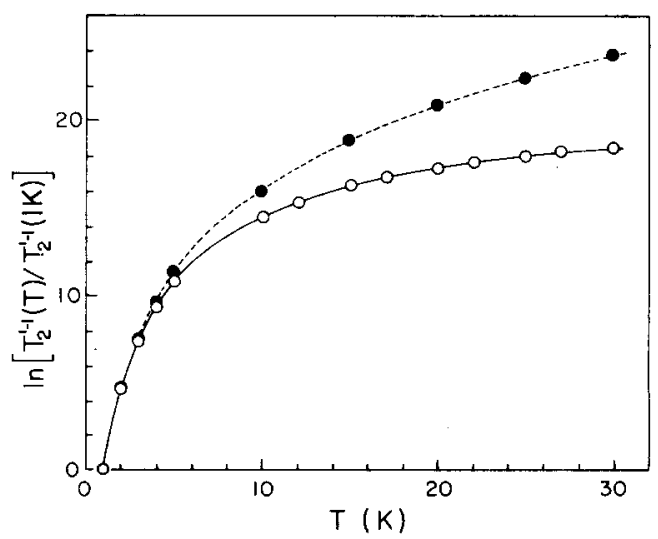

FIG. 2. Plot of $\ln \left[{T_{2}^{\prime \prime}}^{-1}(T) / T_{2}^{\prime-1}\left(1^{\circ} \mathrm{K}\right)\right]$ vs $T$ for Ar. Open circles refer to the "exact" expression (III. 12); closed circles correspond to low-temperature $\left(T^{7}\right)$ approximation. 


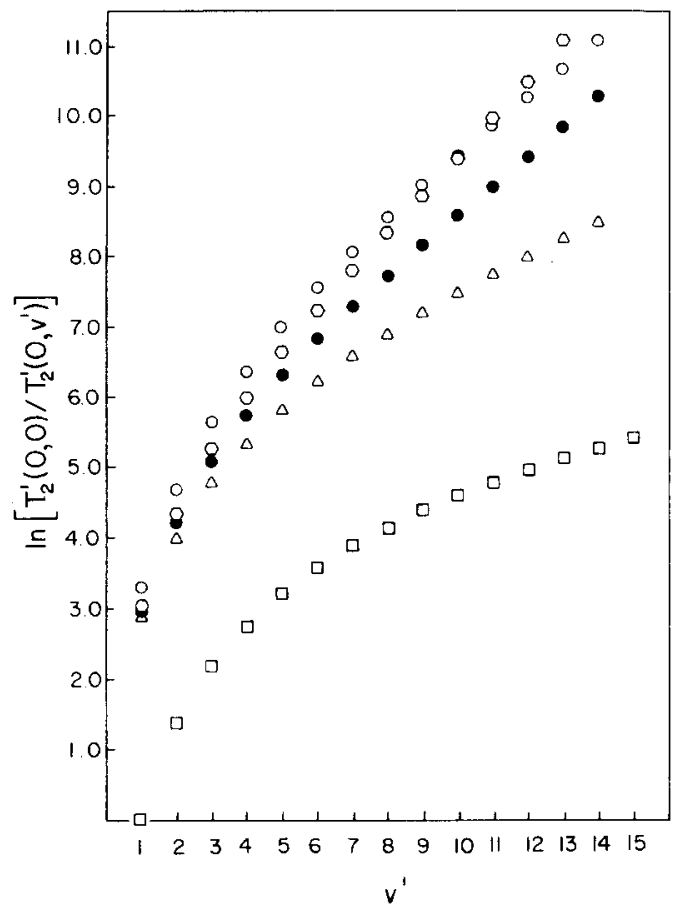

FIG. 3. Plots of $\ln \left[T_{2}^{\prime}(0,0) / T_{2}^{\prime}\left(0, v^{\prime}\right)\right]$ vs $v^{\prime}$ for $\mathrm{Cl}_{2} / \mathrm{Ar}$ using the parameters of Tables I and $I I$, except where indicated otherwise, and various approximations to $v_{i f}: 0$, exact results; $\Delta$, linear approximation to $\phi^{\prime \prime}$ in Eq. (III. 15), i. e., $\phi^{\prime \prime}=$ const. $\times Q ; O$, quadratic approximation to $\phi^{\prime \prime}$, i. e., $\phi^{\prime \prime}(Q)=$ const. $\times Q^{2} ; O$, exact results with $\delta r=1.0 \AA ; \square$, quadratic approximation to $\phi^{\prime \prime}$ and harmonic approximation of $U_{1}^{(t)}$ with identical fundamental frequencies for ground and excited manifolds. Note that in the last case we plot $\ln \left[T_{2}^{\prime}(0,1) / T_{2}^{\prime}\left(0, v^{\prime}\right)\right]=2 \ln v^{\prime}$.

relatively insensitive to variations in $\delta r$ and $\hat{\sigma}$. Plots of $\ln \left[T_{2}^{\prime}(0,0) / T_{2}^{\prime}\left(0, v^{\prime}\right)\right]$ vs $v^{\prime}$ in Fig. 3 demonstrate this point.

\section{Unequal anharmonic couplings}

Since little or no information on excited-state inter molecular potentials (i. e., $\phi_{f}$ ) is available, we have assumed that the excited-state parameters are $\hat{\sigma}$ and $\hat{\epsilon}$ and that the ground state anharmonic coupling vanishes. In Table $\mathrm{V}$, we list $T_{2}^{\prime}$ calculated using the parameters of Table I and taking $\delta r=0$. For purposes of comparison, we also list the $\delta r=0$ column of Table III and the ratio of $\left(T_{2}^{\prime}\right)^{\prime}$ 's calculated in the two extremes. While there is strong disagreement between the calculations for low $-v^{\prime}$ transitions, there is a discrepancy of a factor of only $1-2$ for the high $-v^{\prime}$ transitions. Indeed, we observe a regular decrease toward unity in the ratio of the $\left(T_{2}^{\prime}\right)$ 's as $v^{\prime}$ increases. The reason is that inequality (II. 29) in fact improves with increasing $v^{\prime}$. Thus, the influence of vibrational effects outweighs that of purely electronic effects for sufficiently large $v^{\prime}$. An important corollary is the following: If the anharmonic coupling constant changes significantly in going from the ground to the excited state, then the linewidths of low$v^{\prime}$ transitions will be strongly influenced by purely electronic effects.

\section{COMPARISON WITH OTHER WORK AND CONCLUSIONS}

In this Section, we compare our quantum-mechanical results with the experimental findings ${ }^{3}$ for the system $\mathrm{Cl}_{2} / \mathrm{Ar}$ and with recent theoretical data obtained ${ }^{2}$ using the stochastic-classical-trajectory (SCT) method.

The SCT calculations of Shugard et al. ${ }^{2}$ provide both the dephasing time and the energy-relaxation time under the following assumptions: The lattice is taken to be one dimensional, the diatomic impurity being free to move in the cage effectively formed by the neighboring lattice atoms, which are harmonically bound to fixed equilibrium positions. The potential-energy binding the atoms of the diatomic is taken to be harmonic in the ground state $\left(X^{1} \Sigma_{g}^{+}\right)$and a Morse potential in the excited state $\left(B^{3} \pi_{u}\right)$. The interaction potential between each atom of the diatomic and the nearest lattice atom is assumed to be a repulsive exponential with parameters independent of the electronic state. Since the energy-relaxation time increases markedly with the frequency of the diatomic, Shugard et al. ${ }^{2}$ assume a fundamental frequency of $365 \mathrm{~cm}^{-1}$ (as compared with the observed value of $544 \mathrm{~cm}^{-1}$ ) for the ground-state harmonic binding potential in order to save computer time. Finally, as pointed out by Shugard et al. , ${ }^{2}$ the classical nature of their calculation renders the results at low temperatures, where quantum-mechanical effects may prevail, nonphysical.

In Table VI, we display the results of the SCT computations ${ }^{2}$ together with those obtained (under the same assumption of Ref. 2-equal anharmonic impurity-lattice coupling in the ground and excited electronic states) in the present work. The table also lists the experimental zero-phonon linewidths reported by Bondybey and Fletcher. ${ }^{14}$ From a cursory scan of Table VI, it appears that our calculations of $T_{2}^{\prime}$ using the quantummechanical expression (III. 12) are consistent with the SCT results. However, it should be emphasized that,

TABLE V. $T_{2}^{\prime}$ (psec) for the vibronic transitions $X^{1} \Sigma_{c}^{+}\left(v^{\prime}=0\right) \rightarrow B^{3} \pi_{u}\left(v^{\prime}=0,1, \ldots, 14\right)$ for the system $\mathrm{Cl}_{2} / \mathrm{Ar}$ at $4{ }^{\circ} \mathrm{K}$.

\begin{tabular}{rccc}
\hline \hline$v^{\prime}$ & $T_{2}^{\prime \mathrm{a}}$ & $T_{2}^{\prime \mathrm{b}}$ & $T_{2}^{\prime 2} / T_{2}^{\prime \mathrm{b}}$ \\
\hline 0 & $6.45(5)$ & $6.59(2)$ & 979. \\
1 & $3.36(4)$ & $5.35(2)$ & 62.8 \\
2 & $9.56(3)$ & $4.32(2)$ & 22.1 \\
3 & $4.06(3)$ & $3.47(2)$ & 11.7 \\
4 & $2.07(3)$ & $2.77(2)$ & 7.47 \\
5 & $1.17(3)$ & $2.20(2)$ & 5.32 \\
6 & $7.05(2)$ & $1.73(2)$ & 4.08 \\
7 & $4.42(2)$ & $1.34(2)$ & 3.30 \\
8 & $2.84(2)$ & $1.04(2)$ & 2.73 \\
9 & $1.85(2)$ & $0.79(2)$ & 2.34 \\
10 & $1.21(2)$ & $0.59(2)$ & 2.05 \\
11 & 80.2 & 43.5 & 1.84 \\
12 & 52.5 & 31.4 & 1.67 \\
13 & 34.0 & 22.1 & 1.54 \\
14 & 21.6 & 15.1 & 1.43 \\
\hline \hline
\end{tabular}

${ }^{\mathrm{a}}$ From the $\delta \boldsymbol{r}=0$ column of Table III.

${ }^{\mathrm{b}}$ Calculated under the approximation (II. 29). 
TABLE VI. Comparison of various theoretical and experimental results for the transitions $\left(0, v^{\prime}\right)$ in the system $\mathrm{Cl}_{2} / \mathrm{Ar}$ at $4^{\circ} \mathrm{K}$.

\begin{tabular}{|c|c|c|c|c|}
\hline $\begin{array}{l}\text { Final } \\
\text { vibrational } \\
\text { level } \\
v^{\prime}\end{array}$ & $\begin{array}{l}\text { Energy } \\
\text { relaxation } \\
\text { time, SCT } \\
\text { (psec) }\end{array}$ & $\begin{array}{l}\text { Total } \\
\text { dephasing } \\
\text { time, SCT } \\
\text { (psec) }\end{array}$ & $\begin{array}{l}\text { Pure dephasing } \\
\text { time, present } \\
\text { work }^{2} \\
\text { (psec) }\end{array}$ & $\begin{array}{l}\text { Experimental } \\
\text { results }^{\mathrm{b}}\end{array}$ \\
\hline 5 & 2800 & 12.0 & 16.5 & $\cdots$ \\
\hline 7 & 960 & 6.0 & 6.3 & $\cdots$ \\
\hline 9 & 440 & 2.8 & 2.7 & $\cdots$ \\
\hline 10 & 290 & 2.6 & 1.8 & 1.1 \\
\hline 11 & 340 & 2.4 & 1.2 & 0.9 \\
\hline 12 & $\cdots$ & $\cdots$ & 0.8 & 0.4 \\
\hline 13 & 74 & 1.2 & 0.5 & 0.4 \\
\hline 14 & $\cdots$ & $\cdots$ & 0.3 & 0.3 \\
\hline 15 & 63 & 1.1 & $\cdots$ & $\cdots$ \\
\hline
\end{tabular}

${ }^{\mathrm{a}} \delta r=1.0 \AA, \hat{\sigma}=2.85 \AA$ (see Table $\amalg$ ).

${ }^{b}$ Based on linewidth measurements. See Refs, 2, 3, and 17.

since the SCT method provides the total dephasing time $T_{2}$, whereas our calculation gives $T_{2}^{\prime}$, the results are not strictly comparable, unless energy relaxation (i.e., $T_{1}$ processes) is negligible [see Eq. (I. 1)]. In fact, at low temperatures, where the SCT method is not expected to work well, the effect of $T_{1}$ processes is significant. In other words, the contribution of lifetime broadening to the homogeneous linewidth is nonnegligible compared with $T_{2}^{\prime}$. ${ }^{15}$ Furthermore, at these low temperatures, the trend in our quantum-mechanical results for $T_{2}^{\prime}$ parallels the trend in the observed values more closely than that in the SCT results.

However, in order to compare the calculated and $o b-$ served results directly, one must be assured that the experimental data do indeed reflect the widths of homogeneously broadened lines. In systems ${ }^{16}$ other than $\mathrm{Cl}_{2} / \mathrm{Ar}$, we know that the $(0,0)$ transition is inhomogeneously broadened (homogeneous width/inhomogeneous width $\sim 10^{-3}$ ) while the high-energy $\left(0, v^{\prime}\right)$ transitions are homogeneously broadened (widths $\sim 2 \mathrm{~cm}^{-1}$ ) by fast vibrational relaxation. However, measurement of the apparent width as a function of $v^{\prime}$ is useful only if all $\left(0, v^{\prime}\right)$ transitions are homogeneously broadened. Otherwise, one might incorrectly conclude that the changes in $T_{2}^{\prime}$ with $v^{\prime}$ are quite negligible, since the low-energy transitions are masked by inhomogeneous broadening.

This brings up another point concerning the effect of temperature on $T_{2}^{\prime}$. For a given $\left(0, v^{\prime}\right)$ transition, we expect the linewidth to vary as $T^{2}$ only if $T_{1}$ is negligible. In the presence of fast $T_{1}$ processes, the observed width will depend only slightly on temperature at relatively low temperatures, but quite strongly at relatively higher temperatures at which the $T^{7}$-dependent pure $T_{2}^{\prime}$ process "turns on." The fact that the experimental width for the system $\mathrm{Cl}_{2} / \mathrm{Ar}$ depends only weakly on temperature is consistent with the large contribution of $T_{1}^{-1}$ to the total dephasing rate $T_{2}^{-1}$. However, because of the possible presence of inhomogeneous broadening in the low-energy $\left(0, v^{\prime}\right)$ transitions, an underlying $T^{\gamma}$ or $v^{\prime}$ dependence cannot, in our opinion, ${ }^{17}$ be ruled out unequivocally.
In closing, we note that, in making the above comparison between quantal and classical (SCT) results, we have taken the extreme viewpoint of equal anharmonic coupling in the ground and excited states, as did Shugard et al. ${ }^{2}$ From Table V, we infer that, had we taken the alternate viewpoint, namely, that inequality (II. 29) is valid, our conclusions would not be significantly different, particuarly for the high $v^{\prime}$ transitions.

\section{ACKNOWLEDGMENTS}

We thank the National Science Foundation for generous support of this work. We are also grateful to A. Baba for providing us with enough stimulation.

${ }^{1}$ D. J. Diestler and A. H. Zewail, J. Chem. Phys. 71, 3099 (1979), preceding paper.

${ }^{2}$ (a) M. Shugard, J. C. Tully, and A. Nitzan, J. Chem. Phys. 69, 336 (1978); (b) A. Nitzan, M. Shugard, and J. Tully, J. Chem. Phys. 69, 2525 (1978).

${ }^{3}$ V. E. Bondybey and C. Fletcher, J. Chem. Phys. 64, 3615 (1976).

${ }^{4}$ See, for example, B. DiBartolo, Optical Interactions in Solids (Wiley, New York, 1968).

${ }^{5}$ A. H. Zewail and D. J. Diestler (unpublished). An investigation of the effects on $T_{2}^{{ }^{\prime-1}}$ of employing the full expression (II. 4) is in progress.

${ }^{6}$ Handbook of Mathematical Functions, edited by M. Abramowitz and I. A. Stegun (U.S. GPO, Washington, 1965).

${ }^{7} \mathrm{~K}$. E. Jones and A. H. Zewail, in Advances in Laser Chemistry, edited by A. H. Zewail (Springer, Berlin, 1978), p. 196.

${ }^{8}$ D. J. Diestler, Chem. Phys, Lett. 39, 39 (1976).

${ }^{9}$ P. M. Morse, Phys. Rev. 34, 57 (1929).

${ }^{10}$ See, for example, J. O. Hirschfelder, C. F. Curtiss, and R. Byron Bird, Mnlecular Theory of Gases and Liquids (Wiley, New York, 1954).

${ }^{11}$ To compute $T_{2}^{\prime}$ according to Eq. (II. 19), we need to calculate expectation values of $\phi^{\prime \prime}(Q)$ for the Morse oscillator. However, the second derivative $\phi^{\prime \prime}$ of the $\mathrm{LJ}(12,6)$ potential diverges as $r_{ \pm}^{-14}$ in the limit $r_{ \pm} \rightarrow 0$ and hence the expected values of $\phi^{\prime \prime}$ also diverge. To avoid this divergence, which is an unphysical consequence of the $1 D$ model, we fit the $\operatorname{LJ}(12,6)$ potential to the "softer" Morse form. The reason that we do not employ the Morse form directly is principally one of convenience; there are established procedures for estimating the $\mathrm{LJ}$ parameters approximately.

${ }^{12}$ D. J. Diestler, E. -W. Knapp, and H. Douglas Ladouceur, J. Chem. Phys. 68, 4056 (1978).

${ }^{13} \mathrm{H}$. Douglas Ladouceur and D. J. Diestler, J. Chem. Phys. 70,2620 (1979).

${ }^{14}$ These numbers were not given explicitly in Ref. 3, but were taken from Ref. 2 .

${ }^{15}$ Through a private communication with V. Bondybey, we have learned that in other systems (e.g. $\mathrm{Ca}_{2} / \mathrm{Kr}$ ) the vibrational energy relaxation time is shorter than the emission Iffetime, which suggests the overwhelming influence of $T_{1}$ processes in the upper vibronic levels of such systems.

${ }^{16}$ T. E. Orlowski and A. H. Zewail, J. Chem. Phys. 70, 1390 (1979).

${ }^{17} \mathrm{~V}$. Bondybey has informed us that the temperature dependence of the linewidths quoted in Ref. 2 was determined only very roughly and that more refined and complete measurements are needed. 